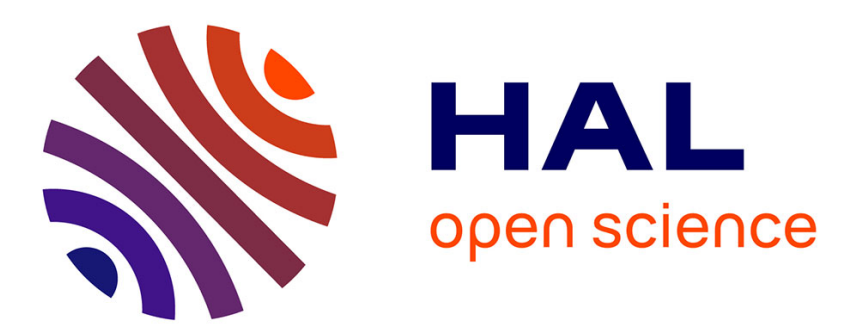

\title{
Porte-échantillon ultra-vide à trois translations et trois rotations pour diffraction d'électrons
}

\author{
Jacques Cazaux, Raymond Ober, Roger Vilanove
}

\section{To cite this version:}

Jacques Cazaux, Raymond Ober, Roger Vilanove. Porte-échantillon ultra-vide à trois translations et trois rotations pour diffraction d'électrons. Revue de Physique Appliquée, 1967, 2 (1), pp.62-64. 10.1051/rphysap:019670020106200 . jpa-00242766

\section{HAL Id: jpa-00242766 https://hal.science/jpa-00242766}

Submitted on 1 Jan 1967

HAL is a multi-disciplinary open access archive for the deposit and dissemination of scientific research documents, whether they are published or not. The documents may come from teaching and research institutions in France or abroad, or from public or private research centers.
L'archive ouverte pluridisciplinaire HAL, est destinée au dépôt et à la diffusion de documents scientifiques de niveau recherche, publiés ou non, émanant des établissements d'enseignement et de recherche français ou étrangers, des laboratoires publics ou privés. 


\title{
PORTE-ÉGHANTILLON ULTRA-VIDE A TROIS TRANSLATIONS ET TROIS ROTATIONS POUR DIFFRAGTION D'ÉLEGTRONS
}

\author{
Par Jagques CAZAUX, Raymond OBER et Roger VIlANOVE, \\ Laboratoire de Physique Théorique, Gollège de France, I I, place Marcelin-Berthelot, Paris (Ve).
}

\begin{abstract}
Résumé. - L'appareil décrit permet de communiquer de l'extérieur trois mouvements de translation et trois mouvements de rotation à un échantillon situé dans une enceinte ultra-vide. Il est actuellement utilisé comme porte-objet dans un appareil de diffraction d'électrons et, dans le cas des mesures par transmission, plusieurs objets peuvent être étudiés successivement.

Abstract. - The apparatus described here allows three translation and three rotary movements of a sample in an ultra-high vacuum chamber. It is in use as crystal holder in an electron diffraction apparatus, and for transmission measurements through polycrystalline materials five specimens may be observed one after another.
\end{abstract}

1. Introduction. - Pour transmettre à un objet placé sous vide trois translations suivant les axes de coordonnées et trois rotations autour de ces mêmes axes centrés sur l'échantillon, nous avons mis au point un porte-échantillon absolument étanche.

Dans son principe, l'appareil représenté figure 1 est constitué d'un bloc assurant les translations, surmonté de l'ensemble des rotations.

2. Appareil. - a) Translations. - Les translations de l'échantillon sont assurées par la déformation d'un seul soufflet en acier inoxydable de $100 \mathrm{~mm}$ de long et de $60 \mathrm{~mm}$ de diamètre extérieur.

Les extrémités du soufflet sont soudées sur des brides : la bride inférieure fixe solidaire de l'enceinte à vide, la bride supérieure solidaire d'une table à glissières constituée de deux plateaux superposés montés chacun sur des rails à galets permettant le déplacement de la bride mobile dans un plan horizontal (l'amplitude de ce déplacement est de $\pm 10 \mathrm{~mm}$ et peut être repérée au 1/10 de $\mathrm{mm}$ ).

L'ensemble de la table ainsi constituée repose sur une plaque montée sur colonnes à billes et peut donc se déplacer verticalement. La commande des translations se fait à l'aide de vis molletées agissant directement sur le plateau relatif au déplacement désiré.

b) Rotations. - La bride supérieure du soufflet des translations est surmontée d'une croix tubulaire sur laquelle viennent s'adapter les rotations. On peut substituer à l'une des rotations un passage de courant destiné à l'alimentation d'un four (afin de chauffer l'échantillon par exemple).
Le principe des rotations est identique et repose sur l'utilisation d'un soufflet légèrement déformé à l'intérieur duquel on a fixé une tige elle-même courbée. On communique de l'extérieur une rotation à l'extrémité excentrée et le mouvement est transmis à la tige centrale sous vide (fig. 2, page 64).

Il faut noter que le système obtenu est rigoureusement étanche (utilisation de joints et de soufflets métalliques), amagnétique, sans glissement (car la transmission du mouvement est intégrale) et étuvable (les différents roulements à billes en acier inoxydable amagnétique pouvant être portés à $200^{\circ} \mathrm{C}$ ).

Le mouvement tournant au sommet de la croix est prolongé par une tige de $200 \mathrm{~mm}$ de long et de $7 \mathrm{~mm}$ de diamètre guidée par des roulements inoxydables et cette tige supporte le porte-échantillon proprement dit en assurant la rotation de Bragg.

La deuxième rotation (rotation azimutale) est fixée sur une branche latérale de la croix et le mouvement est transmis à l'échantillon à l'aide d'un câble en acier inoxydable. De la même manière, l'autre branche latérale peut recevoir la troisième rotation, elle aussi transmise par un câble en acier inoxydable.

c) Le porte-échantillon proprement dit. - Il est constitué d'une roue tangente et d'une vis sans fin actionnée par la rotation azimutale (dans sa version actuelle à deux rotations seulement), l'objet étant fixé au centre de cette roue dentée ( fig. 3, page 64).

On peut adjoindre la troisième rotation, comme le montre la figure $3 b$, à l'aide d'une deuxième roue tangente associée à une deuxième vis sans fin. 


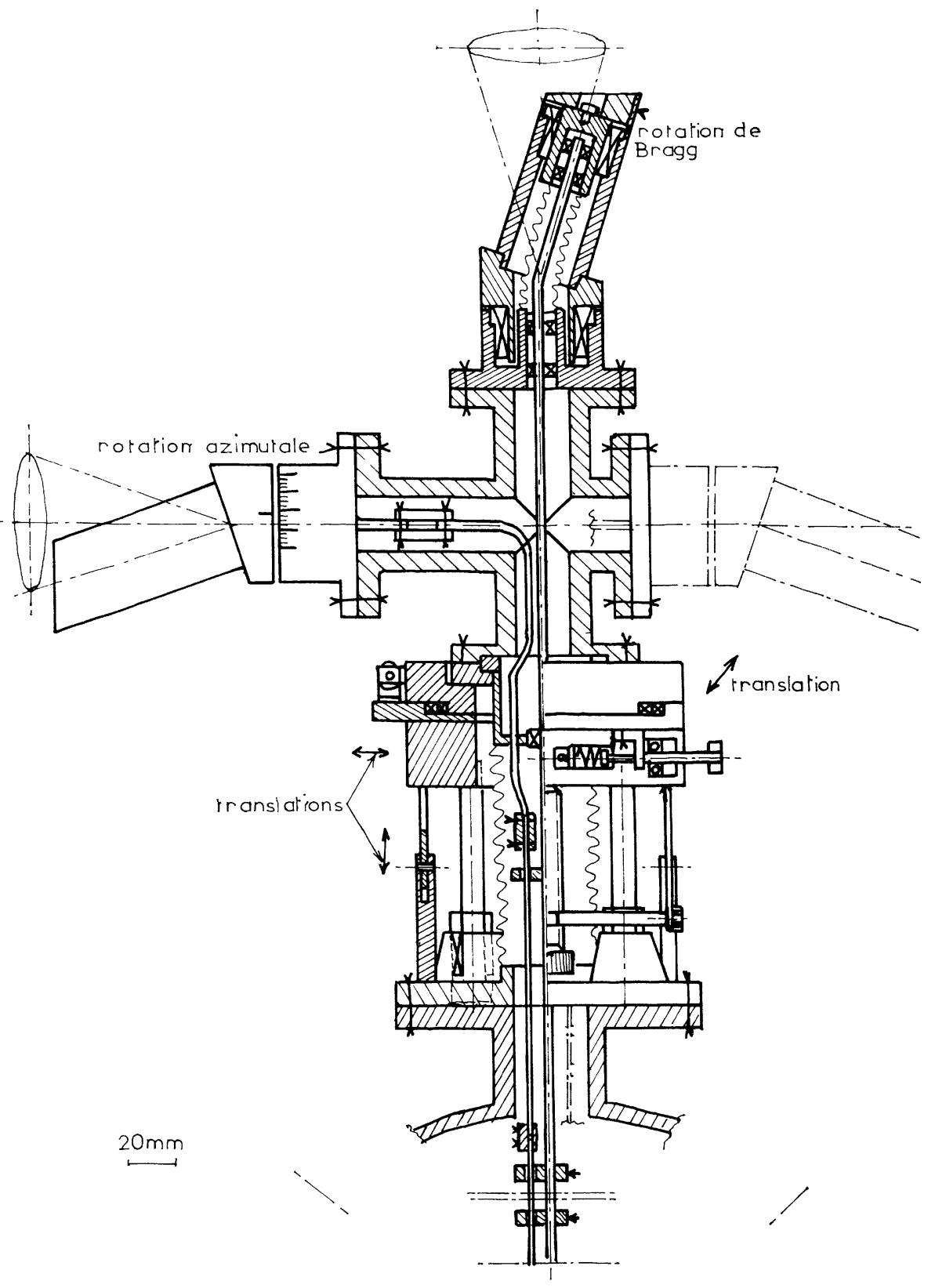

FIG. 1. - Vue d'ensemble du porte-objet.

La rotation de Bragg solidaire de la tige rigide permet une rotation de $360^{\circ}$ sans inconvénient (étant donné la souplesse et la longueur des câbles d'acier assurant les deux autres rotations). Elle peut être repérée avec une précision de l'ordre de 1/10 de degré.

L'amplitude de la rotation azimutale n'est pas limitée ; par contre, étant donné la constante de torsion et la longueur de câble qui communique le mouvement, sa précision et sa fidélité sont limitées à quelques degrés. Il en est de même de la troisième rotation dont l'amplitude est en outre limitée à $\pm 30^{\circ}$ (ce qui est largement suffisant en diffraction d'électrons).

On doit noter que les mouvements ne sont pas indépendants, l'action sur la rotation de Bragg entraînant un mouvement sur les autres rotations, aussi faut-il effectuer les réglages dans l'ordre suivant : rotation de Bragg, troisième rotation, rotation azimutale.

Il est possible de concevoir un système éliminant les inconvénients précités.

Pour améliorer la précision des rotations latérales 


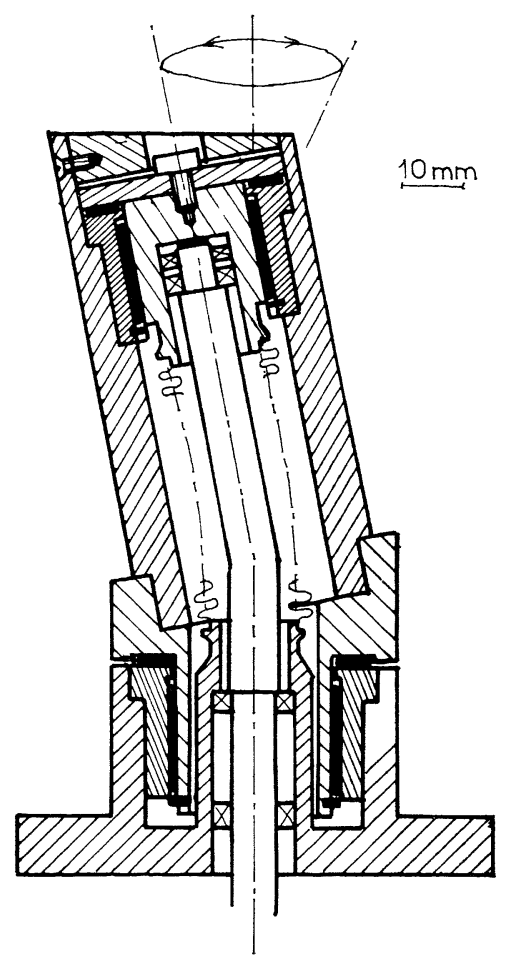

FIG. 2. - Mouvement de rotation. (azimutale et troisième rotation), on peut substituer aux câbles d'acier des transmissions assurées par des tubes d'acier concentriques à la rotation de Bragg et terminés par des pignons coniques.

On peut aussi imaginer le débrayage de ces pignons rendant les trois rotations absolument indépendantes, mais de tels ensembles seraient difficilement étuvables et limiteraient le vide dans l'enceinte.

Notons que, outre l'échantillon placé au centre de la roue dentée assurant la rotation azimutale, on peut disposer de plusieurs autres échantillons sur la périphérie de cette roue afin de les étudier successivement sans faire d'entrée d'air intermédiaire. Dans ce cas, la rotation azimutale permet d'amener les échantillons successifs devant le faisceau d'électrons.

3. Conclusion. - L'appareil décrit, de conception et de réalisation très simples, permet d'étudier en diffraction d'électrons plusieurs échantillons successivement et de communiquer à l'un d'entre eux trois translations et trois rotations en ultra-vide.

Il présente l'avantage de n'immobiliser qu'une seule bride de l'enceinte sur laquelle il est monté et si son encombrement est assez important, le volume occupé au voisinage de l'objet est réduit au minimum.

Manuscrit reçu le 6 janvier 1967.

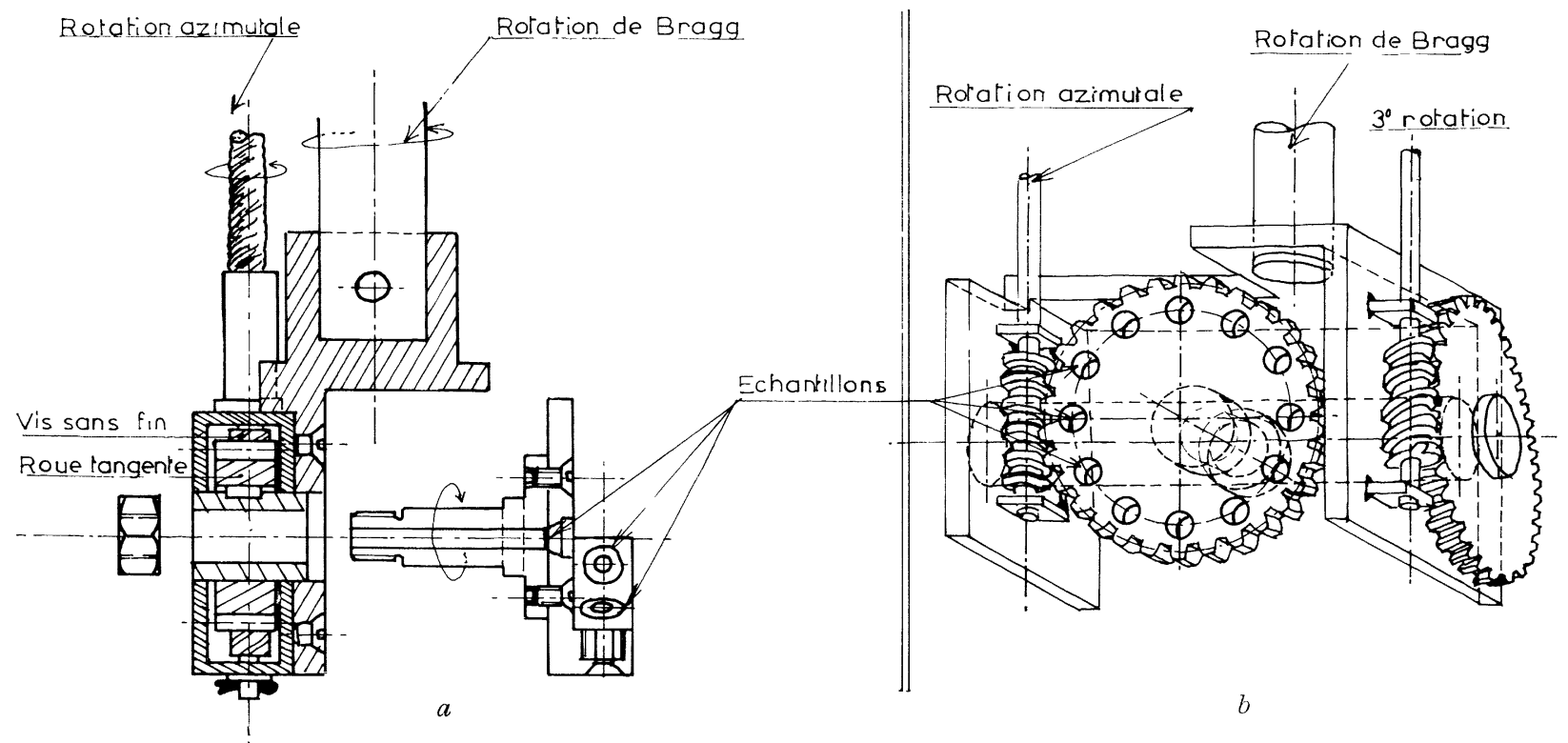

FIG. 3. - Porte-échantillon proprement dit

a) Modèle à 2 rotations ; b) Modèle à 3 rotations.

Le Directeur de la Publication: Jules-Jean Chovin. - Imprimerie des Presses Universitaires de France, Vendôme (France) Dépôt légal : 2-1967 